\title{
Novel Techniques in Endoscopy Are Useful in Evaluating Patients with Ulcerative Colitis
}

\author{
Takafumi Ando, ${ }^{1}$ Osamu Watanabe, ${ }^{1}$ Yuji Nishio, ${ }^{2}$ Kazuhiro Ishiguro, ${ }^{1}$ Osamu Maeda, ${ }^{1}$ \\ Masanao Nakamura, ${ }^{1}$ Ryoji Miyahara, ${ }^{1}$ Naoki Ohmiya, ${ }^{1}$ and Hidemi Goto ${ }^{1}$ \\ ${ }^{1}$ Department of Gastroenterology, Nagoya University Graduate School of Medicine, 65 Tsurumai-cho, Showa-ku, Nagoya City, \\ Aichi 466-8550, Japan \\ ${ }^{2}$ Department of Gastroenterology, Meitetsu Hospital, 2-26-11 Sako, Nishi-ku, Nagoya City, Aichi 451-8511, Japan
}

Correspondence should be addressed to Takafumi Ando, takafumiando-gi@umin.ac.jp

Received 30 August 2010; Revised 29 March 2011; Accepted 15 April 2011

Academic Editor: Hajime Kuwayama

Copyright ( $) 2011$ Takafumi Ando et al. This is an open access article distributed under the Creative Commons Attribution License, which permits unrestricted use, distribution, and reproduction in any medium, provided the original work is properly cited.

Ulcerative colitis (UC), one of the most common forms of chronic inflammatory bowel disease, is characterized by exacerbations and remissions. Even when conventional colonoscopy suggests remission and a normal mucosal finding, microscopic or histological abnormalities may persist, and relapse may be imminent. Confocal microendoscopy allows histological diagnosis during the endoscopic examination. High-resolution video-magnifying colonoscopy with chromoscopy enables the observation of colorectal mucosal pit patterns and prediction of the probability of subsequent disease relapse in patients in remission. Endoscopic ultrasonography provides an immediate and accurate evaluation of the health status of the colonic wall without the need to wait for histological results and provides an indication of the efficacy of treatment. These novel endoscopic techniques are useful for the evaluation of disease activity and the efficacy of treatment in patients with UC and the prediction of relapse.

\section{Background}

Ulcerative colitis (UC) is a chronic inflammatory bowel disorder which shows evidence of activation of the immune system of the colorectum, with exacerbations and remissions [1-5]. Disease activity in UC patients is usually assessed by clinical observation, laboratory data, and conventional colonoscopic examination, which allows superficial changes in the colonic mucosa to be discerned. For example, a combination of three simple clinical or biological criteria, namely, tachycardia, fever, and CRP, is useful in predicting response to cyclosporine [6], and conventional colonoscopy is important in evaluating the severity and extent of UC and in the selection of treatments for patients with UC [7]. However, although the degree of inflammation as assessed by routine colonoscopy is a reliable parameter of disease activity, discrepancies between colonoscopic appearance and histopathologic abnormalities are sometimes seen in patients with clinically inactive disease. Even when routine colonoscopy suggests remission and a normal mucosal appearance, microscopic abnormalities may persist $[8,9]$ and relapse may be imminent. Riley et al. [10] reported that among 82 UC patients with symptomatic and endoscopic remission, 27 (33\%) relapsed during the 12-month followup. Relapse rates were unrelated to the duration or extent of disease or the type of maintenance drug treatment. In patients with an acute inflammatory cell infiltrate 52\% relapsed, whereas in the absence of such an infiltrate only $25 \%$ relapsed $(P=002)$. Various techniques which enable the diagnosis of many types of gastrointestinal lesions have been developed, such as magnifying endoscopy and chromoendoscopy $[11,12]$, which enables the diagnosis of many types of gastrointestinal lesions. A recently developed highresolution video-magnifying colonoscope has enabled the observation of pit patterns on the surface of the colorectal mucosa. This in turn allows an understanding of the morphological relationship between the pit patterns detected colonoscopically and crypts observed histopathologically [13-17]. Confocal microscopy is a technique that provides clear microscopic images by using small apertures [18]. 
Laser light that enters through an illumination aperture is focused on a sample by the objective lens, and excitation light given off by a fluorescent dye passes through a confocal aperture and is converted into an electrical signal by a detector. At points away from the focal plane, the excitation light is weak and also cannot pass through the confocal aperture. Because signals from out-of-focus areas are thus doubly weakened, this allows selective detection of signals from the focal plane alone. Confocal microscopy allows the observation of living cells in vivo and histological examination of the mucosal layer, which can be done at the time of colonoscopy to enhance diagnostic precision and guide subsequent therapeutic strategies. A recent report [19] in 83 Italian patients with moderate to severe UC assessed by clinical measures, endoscopic criteria, as well as US showed that both US and endoscopy were more predictive of disease outcome than clinical criteria. Recent technical progress in endoscopic ultrasonography (EUS) has facilitated the acquisition of information about the transmural structure of the bowel wall in UC patients even more precisely [20-24]. Because EUS findings correlate with histological findingsan increased number of cells infiltrating the lamina propria result in an increased mucosal thickness-EUS can be used to objectively evaluate the degree of vertical spread of intestinal inflammation in UC. EUS also has been reported to be useful in predicting the occurrence of relapse in UC patients in remission [22].

Here, we discuss confocal endomicroscopy, a novel endoscopy technique which is predictive of the efficacy of therapies in patients with UC, endoscopic factors in magnifying colonoscopy that may predict the probability of subsequent disease relapse in UC patients in remission, and the usefulness of EUS as a diagnostic technique to assess disease status. We also reconsider the value of endoscopy in the treatment of patients with UC.

\section{Literature Review}

2.1. Histologic Assessment by Confocal Endomicroscopy in Patients with Ulcerative Colitis. Definitive diagnosis using conventional endoscopy requires the histologic examination of a targeted biopsy, but this can take a number of days and carries the risk of bleeding. The ability to make a definitive diagnosis during endoscopy without biopsy would be useful. Confocal endomicroscopy, a recently developed method which provides high-magnification images of the gastrointestinal epithelium [25], is primarily characterized by its ability to obtain images of cellular and subcellular structures of the gastrointestinal mucosa during endoscopic examination. The aim of this technique is to develop a tool that can offer "virtual" histology diagnosis without the need to take biopsy samples. This method has been used to examine neoplastic lesions in the upper and lower gastrointestinal tract $[18,26-30]$ and in UC has been shown to provide information equivalent to conventional histology (Figure 1) [31]. Confocal endomicroscopy is also useful for predicting hypervascularization as an activity parameter in patients with UC. Neumann et al. [32] reported that confocal endomicroscopy clearly demonstrated hypervascularization and vessel leakiness in patients with UC. Tarnawski et al. [33] reported that confocal endomicroscopy demonstrated impaired mucosal regeneration, persistent inflammation, abnormal microvasculature, and increased vascular permeability in vivo in normal appearing colonic mucosa of patients with UC in remission. To our knowledge, the association of the changes detected by confocal microscopy with increasing risk of relapse has not been reported. However, confocal endomicroscopy is considered a promising diagnostic tool that will allow "virtual" histology and has the potential to monitor patients with UC, even in those with macroscopically normal mucosa.

\subsection{Assessment by Magnifying Colonoscopy Predicts Relapse in} Patients with Quiescent Ulcerative Colitis. Various attempts have been made over many years to observe the magnified features of the gastrointestinal mucosa by stereomicroscopy of resected specimens. In their 1980 stereomicroscopy study of biopsy specimens from the rectal mucosa of UC patients, Poulsen et al. [34] identified microstructural abnormalities on the mucosal surface in almost every patient as well as a close correlation between stereomicroscopic features and clinical disease activity, sigmoidoscopic findings, and histologic activity of the disease. In particular, they compared colonoscopic findings in UC patients with stereomicroscopic findings in biopsy specimens and found a close correlation between the number of crypt openings and clinical disease activity. Recent advances in high-resolution videomagnifying colonoscopy have enabled observation of the surface microstructure of colorectal mucosa, with results approaching the diagnostic capability of the stereomicroscope [35]. The diagnostic ability of magnifying colonoscopy in histological disease activity has been confirmed in patients with both inactive and active UC. Furthermore, use of the high-resolution video colonoscope facilitates the differentiation of quiescent from inflamed mucosa $[15,36-$ 38]. Moreover, it is not unusual for routine colonoscopy performed to assess UC stage to show quiescent colitis despite the histological persistence of inflammation $[9,10$, 39], which later results in the clinical relapse [10]. Again, the high-resolution video colonoscope may allow in vivo optical biopsy so as to guide subsequent therapeutic strategies.

We performed magnifying colonoscopy (MCS) in 113 patients with ulcerative colitis in remission [40] and evaluated the relationship between pit patterns and histological disease activity. The term "pit" refers to the orifices of the colorectal crypts, and the "pit pattern" is the specific arrangement of the opening of an individual crypt. In UC patients, pit pattern may be influenced by the process of destruction and regeneration of glandular ducts. We classified "pit patterns" in the rectal mucosa into four MCS grades (grade $1, n=19$; grade $2, n=44$; grade 3, $n=35$; grade $4, n=15$ ) on the basis of size, shape, and arrangement (Figure 2). Histological disease activity was found to increase with increasing MCS grade $(P=$ .0001). Follow-up for at least 12 months after the start of the study was possible in all patients. During the study, 33 


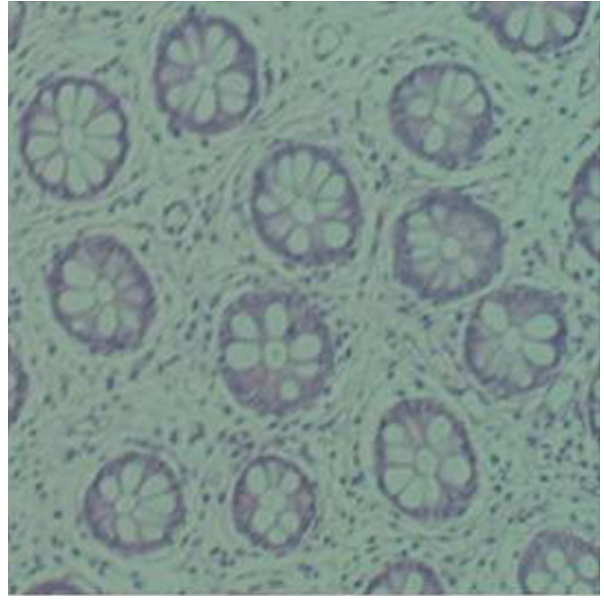

(a)

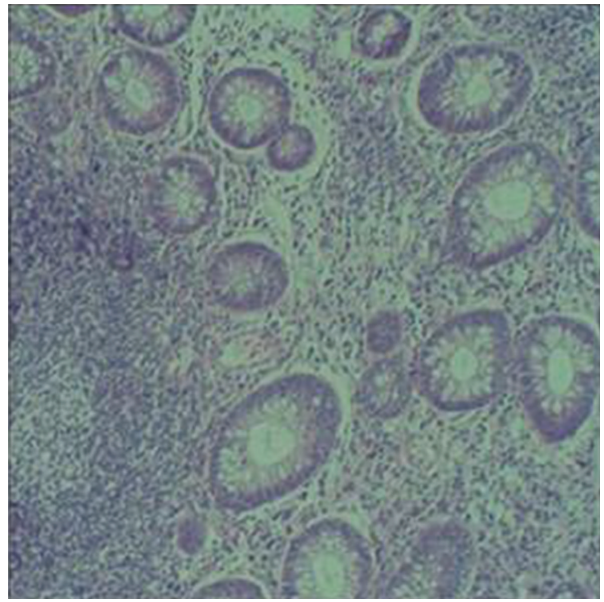

(c)

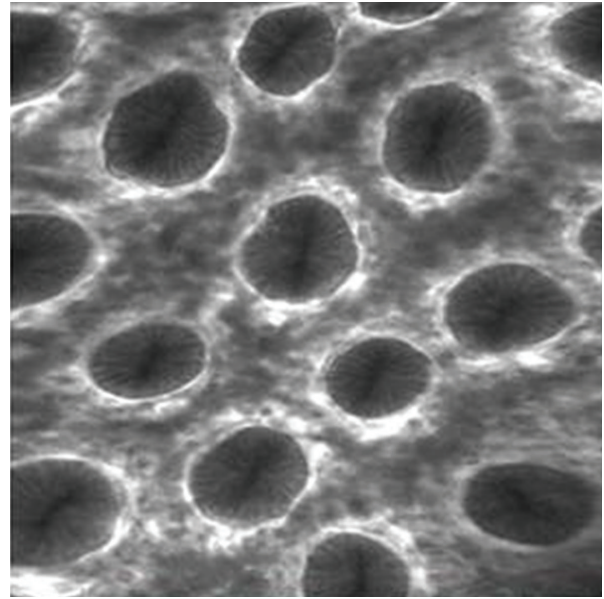

(b)

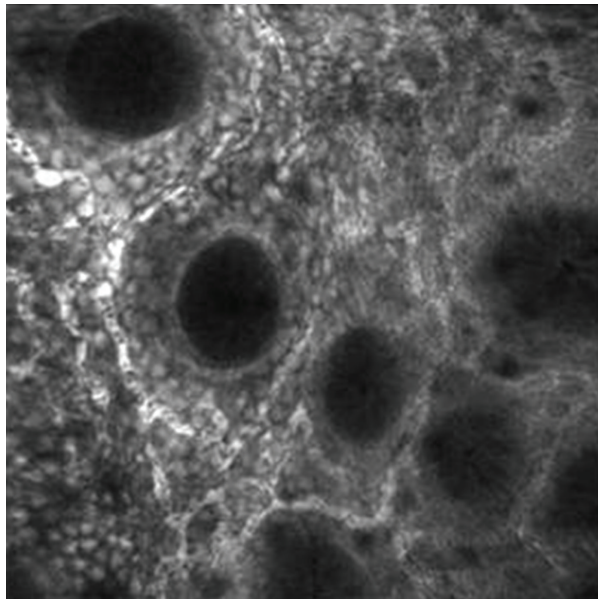

(d)

FIGURE 1: Conventional transverse section of normal (a) and inflamed mucosa (c) and confocal image of normal (b) and inflamed mucosa (d). The confocal image correlates well with the histologic image.

(29.2\%) patients relapsed, with a mean time to relapse of 6.8 months (range, 1-12 months). When hazard ratios, 95\% confidence intervals (CIs), and $P$-values for clinical profile, MCS grade, hematological parameters, mucosal IL-8 activity, and histological grade were calculated, statistically significant associations with relapse were seen for WBC (relative risk: $1.14,95 \%$, CI: $1.00-1.29, P=.047)$, the hazard ratios of MCS grades (relative risk: 2.31, 95\%, CI: 1.56-3.41, $P<.0001$ ), and mucosal IL- 8 activity (relative risk: $1.01,95 \%, \mathrm{CI}: 1.00-$ $1.01, P=.004)$. In contrast, no influence on relapse was seen for clinical profile, ESR, CRP, or histological grade. Multivariate analysis revealed a significant relation between MCS grade and relapse (relative risk: $2.06,95 \%, \mathrm{CI}: 1.34$ $3.17, P=.0009$ ), but not between relapse and $\mathrm{WBC}$ or mucosal IL- 8 activity. The Kaplan-Meier estimate of relapse during 12-month follow-up was found to increase with increasing MCS grade, with percentages of $0 \%(0 / 19)$ for grade $1,21 \%$ (9/44) for grade 2, 43\% (15/35) for grade 3 , and $60 \%$ (9/15) for grade 4 .

Recent studies [41-45] have shown that cytokine measurement can detect subtle but ongoing escalation of the inflammatory process in the mucosa of patients with inflammatory bowel disease (IBD) before clinical symptoms and endoscopic inflammation are apparent. IL-8, a member of the chemokine subfamily, attracts and activates primarily neutrophils, and elevation of IL-8 activity is in fact a wellknown phenomenon in UC patients $[46,47]$. Yamamoto et al. [45] reported that mucosal Il-8 levels predicted future exacerbations of UC. We examined the association between mucosal pit patterns as assessed by magnifying colonoscopy and mucosal IL-8 activity and also evaluated whether this chemokine is able to predict clinical relapse in UC patients [40]. Results showed that mucosal IL-8 activity correlated positively with MCS grade, but that was a less useful predictor of clinical relapse than MCS grade. Moreover, although MCS grade correlated positively with histological grade and mucosal IL- 8 activity, these latter two parameters were less accurate predictors of disease relapse. MCS allows the observation of a wider and more representative area of the colorectal mucosa than biopsy specimens, and MCS grading may be a more accurate predictor of clinical relapse than histological grading. 


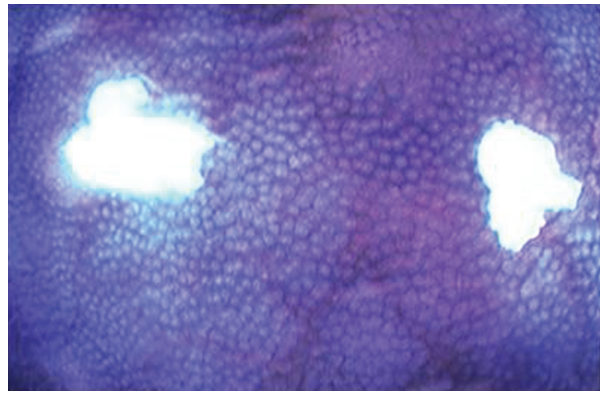

(a)

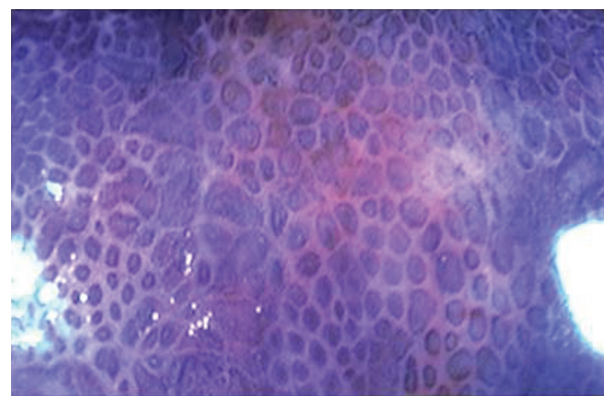

(c)

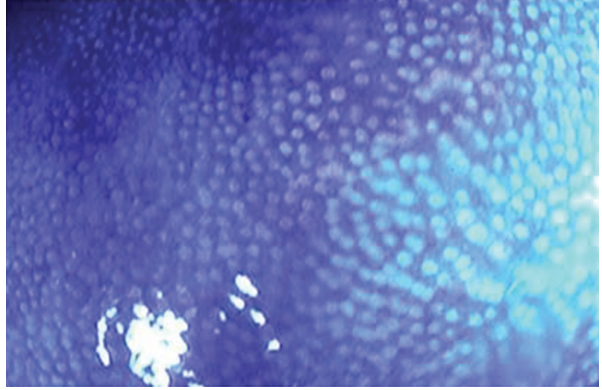

(b)

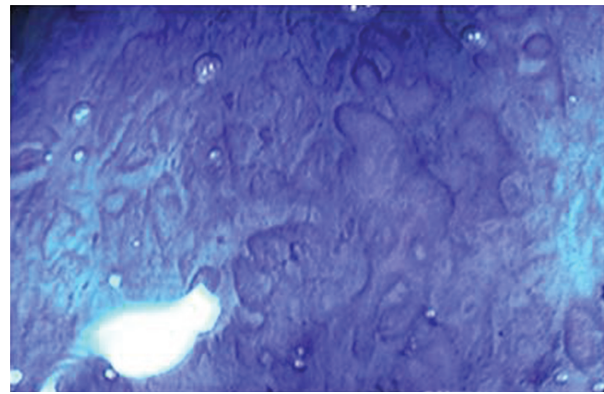

(d)

FIGURE 2: Grading of pit structures in the colorectal mucosa of patients with inactive UC. MCS grade 1: pits small, round, and regularly arranged (a). MCS grade 2: pits rather large, oval, and somewhat irregular in arrangement (b). MCS grade 3: pits of various shapes and sizes and irregularly arranged (c). MCS grade 4: dispersed pits varying in morphology associated with the presence of small erosions (d).

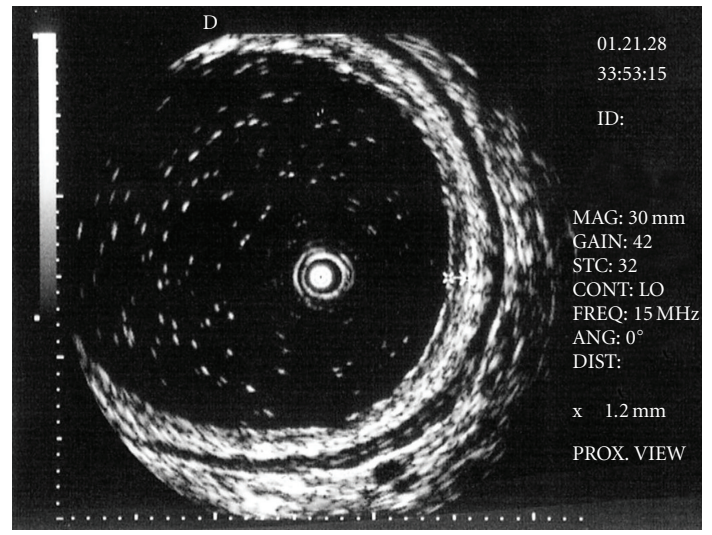

FIGURE 3: Evaluation of thickness of the mucosal layer using an ultrasonic catheter probe at the Rs portion. Thickness of the mucosal layer in this UC patient is $1.2 \mathrm{~mm}$.

2.3. Role of Endoscopic Ultrasonography in Predicting the Response to Cyclosporin A in Ulcerative Colitis Refractory to Steroids. In acute exacerbation of UC, histological findings show diffuse infiltration of neutrophils with accumulation inside and around the crypt epithelium of the affected mucosa $[48,49]$. Recent improvements in ultrasonography technology, particularly the development of ultrasonic catheter miniature probes, have made possible the simple and detailed evaluation of the colorectal wall structure, and several ultrasonic catheter probe techniques for the assessment of disease activity in UC patients have been introduced [22, 23]. Using this technique, Hurlstone et al. [23] reported that mucosa and submucosa were significantly thicker in Matts' histopathological grade [8] 3b and 4 disease than in earlier Matts' grades. In their 1-year prospective study of patients with UC who were receiving remission maintenance therapy [22], Higaki et al. reported that the thickness of the first to the third layer of the rectal wall, as evaluated by EUS at the beginning of the study, was significantly greater in the relapse group than that in the nonrelapse group, which demonstrated the usefulness of EUS in predicting the occurrence of clinical relapse of UC. We reported [50] a significant correlation between histological grading as determined by Riley's score and thickness of the rectal mucosa as determined using an ultrasonic catheter probe (Figure 3), which in turn suggested that the thickness of the rectal mucosa may reflect the intensity of histological inflammation. EUS is also effective for predicting the response to therapies in UC. We treated 15 UC patients who did not respond to high doses of corticosteroids with CsA. Colonoscopy and EUS were undertaken before and 20 days after CsA therapy. Following treatment with CsA, nine patients showed a decrease in CAI score by six points or more and were defined as responders, while the other six were defined as nonresponders. EUS measurement using an ultrasonic catheter probe showed that thickness of the rectal mucosal layer before CsA was significantly greater in responders than that in nonresponders $(P<.05)$. We found that the ultrasonic catheter probe was a useful means of predicting and evaluating the efficacy of CsA treatment in severely ill UC patients. In this study, EUS was done by a single 
endoscopist who is specialized in this procedure; however, for generalization of EUS assessment, interobserver variability among endoscopists should be analysed. Although further investigation remains to be done, including correlations with the efficacy of other therapies such as steroids, immunesuppressive agents, and leukocytapheresis, this method is a promising technique for the assessment of disease status and direct therapeutic management.

\section{Discussion and Conclusions}

In this paper, we evaluated the potential application of three new endoscopic techniques, confocal endomicroscopy, magnifying colonoscopy, and EUS, in UC patients. These techniques highlight the frequent discrepancy between macroscopic and microscopic findings in UC. Early reports that these imaging modalities may play an important role in the assessment and medical management of UC patients are encouraging. However, the presence of variability among endoscopists in interpreting these specialized images should be kept in mind as most reports have not considered this important point. When we consider the application of these novel techniques to Crohn's disease (CD) patients, diagnostic observation of the magnified features of the gastrointestinal mucosa may not be particularly useful, because CD is a transmural disease. However, EUS in CD patients is useful in the assessment of perianal lesions [51], and the combined use of EUS in combination medical and surgical therapy for perianal CD improves outcomes. Novel endoscopic techniques are useful in the evaluation of disease activity and prediction of clinical relapse in patients with UC, and in the detailed evaluation of the efficacy of treatment. However, because the published literature on this topic is small, the present encouraging preliminary findings will have to be confirmed in larger, more rigorous studies to show the advantage of these new endoscopic methods over currently available disease activity assessment tools.

\section{References}

[1] J. K. Ritchie, J. Powell-Tuck, and J. E. Lennard-Jones, "Clinical outcome of the first ten years of ulcerative colitis and proctitis," Lancet, vol. 1, no. 8074, pp. 1140-1143, 1978.

[2] E. Langholz, P. Munkholm, M. Davidsen, and V. Binder, "Course of ulcerative colitis: analysis of changes in disease activity over years," Gastroenterology, vol. 107, no. 1, pp. 3-11, 1994.

[3] F. C. Edwards and S. C. Truelove, "The course and prognosis of ulcerative colitis," Gut, vol. 4, pp. 299-315, 1963.

[4] W. Selby, "The natural history of ulcerative colitis," Bailliere's Clinical Gastroenterology, vol. 11, no. 1, pp. 53-64, 1997.

[5] N. Hiwatashi, T. Yao, H. Watanabe et al., "Long-term followup study of ulcerative colitis in Japan," Journal of Gastroenterology, vol. 30, supplement 8, pp. 13-16, 1995.

[6] W. Cacheux, P. Seksik, M. Lemann et al., "Predictive factors of response to cyclosporine in steroid-refractory ulcerative colitis," American Journal of Gastroenterology, vol. 103, no. 3, pp. 637-642, 2008.

[7] M. Naganuma, H. Ichikawa, N. Inoue et al., "Novel endoscopic activity index is useful for choosing treatment in severe active ulcerative colitis patients," Journal of Gastroenterology, vol. 45, no. 9, pp. 936-943, 2010.

[8] S. G. Matts, "The value of rectal biopsy in the diagnosis of ulcerative colitis," The Quarterly Journal of Medicine, vol. 30, pp. 393-407, 1961.

[9] J. Powell-Tuck, D. W. Day, and N. A. Buckell, "Correlations between defined sigmoidoscopic appearances and other measures of disease activity in ulcerative colitis," Digestive Diseases and Sciences, vol. 27, no. 6, pp. 533-537, 1982.

[10] S. A. Riley, V. Mani, M. J. Goodman, S. Dutt, and M. E. Herd, "Microscopic activity in ulcerative colitis: what does it mean?" Gut, vol. 32, no. 2, pp. 174-178, 1991.

[11] A. Ohashi, Y. Niwa, N. Ohmiya et al., "Quantitative analysis of the microvascular architecture observed on magnification endoscopy in cancerous and benign gastric lesions," Endoscopy, vol. 37, no. 12, pp. 1215-1219, 2005.

[12] T. Okabayashi, T. Gotoda, H. Kondo et al., "Usefulness of indigo carmine chromoendoscopy and endoscopic clipping for accurate preoperative assessment of proximal gastric cancer," Endoscopy, vol. 32, no. 10, p. S62, 2000.

[13] S. Kudo, C. A. Rubio, C. R. Teixeira, H. Kashida, and E. Kogure, "Pit pattern in colorectal neoplasia: endoscopic magnifying view," Endoscopy, vol. 33, no. 4, pp. 367-373, 2001.

[14] S. Kato, T. Fujii, I. Koba et al., "Assessment of colorectal lesions using magnifying colonoscopy and mucosal dye spraying: can significant lesions be distinguished?" Endoscopy, vol. 33, no. 4, pp. 306-310, 2001.

[15] R. Kiesslich, J. Fritsch, M. Holtmann et al., "Methylene blueaided chromoendoscopy for the detection of intraepithelial neoplasia and colon cancer in ulcerative colitis," Gastroenterology, vol. 124, no. 4, pp. 880-888, 2003.

[16] D. P. Hurlstone, S. S. Cross, I. Adam et al., "Efficacy of high magnification chromoscopic colonoscopy for the diagnosis of neoplasia in flat and depressed lesions of the colorectum: a prospective analysis," Gut, vol. 53, no. 2, pp. 284-290, 2004.

[17] D. P. Hurlstone and S. S. Cross, "Role of aberrant crypt foci detected using high-magnification-chromoscopic colonoscopy in human colorectal carcinogenesis," Journal of Gastroenterology and Hepatology, vol. 20, no. 2, pp. 173-181, 2005.

[18] S. Kitabatake, Y. Niwa, R. Miyahara et al., "Confocal endomicroscopy for the diagnosis of gastric cancer in vivo," Endoscopy, vol. 38, no. 11, pp. 1110-1114, 2006.

[19] F. Parente, M. Molteni, B. Marino et al., "Are colonoscopy and bowel ultrasound useful for assessing response to short-term therapy and predicting disease outcome of moderate-to-severe forms of ulcerative colitis: a prospective study," American Journal of Gastroenterology, vol. 105, no. 5, pp. 1150-1157, 2010.

[20] U. Hildebrandt, J. Kraus, K. W. Ecker, T. Schmid, G. Schuder, and G. Feifel, "Endosonographic differentiation of mucosal and transmural nonspecific inflammatory bowel disease," Endoscopy, vol. 24, supplement 1, pp. 359-363, 1992.

[21] A. M. Soweid, A. Chak, J. A. Katz, and M. Sivak, "Catheter probe-assisted endoluminal US in inflammatory bowel disease," Gastrointestinal Endoscopy, vol. 50, no. 1, pp. 41-46, 1999.

[22] S. Higaki, H. Nohara, Y. Saitoh et al., "Increased rectal wall thickness may predict relapse in ulcerative colitis: a pilot follow-up study of ultrasonographic colonoscopy," Endoscopy, vol. 34, no. 3, pp. 212-219, 2002.

[23] D. P. Hurlstone, D. S. Sanders, A. J. Lobo, M. E. McAlindon, and S. S. Cross, "Prospective evaluation of high-frequency mini-probe ultrasound colonoscopic imaging in ulcerative 
colitis: a valid tool for predicting clinical severity," European Journal of Gastroenterology and Hepatology, vol. 17, no. 12, pp. 1325-1331, 2005.

[24] S. Yoshizawa, K. Kobayashi, T. Katsumata, K. Saigenji, and I. Okayasu, "Clinical usefulness of EUS for active ulcerative colitis," Gastrointestinal Endoscopy, vol. 65, no. 2, pp. 253-260, 2007.

[25] A. L. Polglase, W. J. McLaren, S. A. Skinner, R. Kiesslich, M. F. Neurath, and P. M. Delaney, "A fluorescence confocal endomicroscope for in vivo microscopy of the upper- and the lower-GI tract," Gastrointestinal Endoscopy, vol. 62, no. 5, pp. 686-695, 2005.

[26] Y. Kakeji, S. Yamaguchi, D. Yoshida et al., "Development and assessment of morphologic criteria for diagnosing gastric cancer using confocal endomicroscopy: an ex vivo and in vivo study," Endoscopy, vol. 38, no. 9, pp. 886-890, 2006.

[27] R. Kiesslich, A. Hoffman, M. Goetz et al., "In vivo diagnosis of collagenous colitis by confocal endomicroscopy," Gut, vol. 55, no. 4, pp. 591-592, 2006.

[28] R. Kiesslich, J. Burg, M. Vieth et al., "Confocal laser endoscopy for diagnosing intraepithelial neoplasias and colorectal cancer in vivo," Gastroenterology, vol. 127, no. 3, pp. 706-713, 2004.

[29] R. Kiesslich, M. Goetz, M. Vieth, P. R. Galle, and M. F. Neurath, "Confocal laser endomicroscopy," Gastrointestinal Endoscopy Clinics of North America, vol. 15, no. 4, pp. 715-731, 2005.

[30] A. Hoffman, M. Goetz, M. Vieth, P. R. Galle, M. F. Neurath, and R. Klesslich, "Confocal laser endomicroscopy technical status and current indications," Endoscopy, vol. 38, no. 12, pp. 1275-1283, 2006.

[31] O. Watanabe, T. Ando, O. Maeda et al., "Confocal endomicroscopy in patients with ulcerative colitis," Journal of Gastroenterology and Hepatology, vol. 23, supplement 2, pp. S286S290, 2008.

[32] H. Neumann, M. Vieth, R. Atreya et al., "Hypervascularization as an activity parameter in patients with ulcerative colitis diagnosed by confocal laser endomicroscopy," Gut, vol. 59, supplement 3, p. A37, 2010.

[33] A. S. Tarnawski, E. Coron, Z. Sandor et al., "In-vitro cellular and molecular imaging of mucosal healing quality in ulcerative colitis in remission shows impaired crypt regeneration, pathological angiogenesis and persistent inflammation: new molecular mechanisms include aberrant survivin and VEGF," Gut, vol. 59, supplement 3, p. A400, 2010.

[34] S. S. Poulsen, K. C. Christensen, M. Petri, and S. Jarnum, "Stereomicroscopic examination of stained rectal biopsies in ulcerative colitis and Crohn's disease," Scandinavian Journal of Gastroenterology, vol. 15, no. 5, pp. 535-544, 1980.

[35] S. Kudo, S. Hirota, T. Nakajima et al., "Colorectal tumours and pit pattern," Journal of Clinical Pathology, vol. 47, no. 10, pp. 880-885, 1994.

[36] T. Matsumoto, F. Kuroki, M. Mizuno, S. Nakamura, and M. Iida, "Application of magnifying chromoscopy for the assessment of severity in patients with mild to moderate ulcerative colitis," Gastrointestinal Endoscopy, vol. 46, no. 5, pp. 400-405, 1997.

[37] M. Fujiya, Y. Saitoh, M. Nomura et al., "Minute findings by magnifying colonoscopy are useful for the evaluation of ulcerative colitis," Gastrointestinal Endoscopy, vol. 56, no. 4, pp. 535-542, 2002.

[38] M. Kunihiro, S. Tanaka, M. Sumii et al., "Magnifying colonoscopic features of ulcerative colitis reflect histologic inflammation," Inflammatory Bowel Diseases, vol. 10, no. 6, pp. 737-744, 2004.
[39] C. G. Kleer and H. D. Appelman, "Ulcerative Colitis: patterns of involvement in colorectal biopsies and changes with time," American Journal of Surgical Pathology, vol. 22, no. 8, pp. 983989, 1998.

[40] Y. Nishio, T. Ando, O. Maeda et al., "Pit patterns in rectal mucosa assessed by magnifying colonoscope are predictive of relapse in patients with quiescent ulcerative colitis," Gut, vol. 55, no. 12, pp. 1768-1773, 2006.

[41] S. Schreiber, S. Nikolaus, J. Hampe et al., "Tumour necrosis factor $\alpha$ and interleukin $1 \beta$ in relapse of Crohn's disease," Lancet, vol. 353, no. 9151, pp. 459-461, 1999.

[42] I. D. R. Arnott, H. E. Drummond, and S. Ghosh, "Gut mucosal secretion of interleukin $1 \beta$ and interleukin- 8 predicts relapse in clinically inactive Crohn's disease," Digestive Diseases and Sciences, vol. 46, no. 2, pp. 402-409, 2001.

[43] B. Meresse, P. Rutgeerts, H. Malchow et al., "Low ileal interleukin 10 concentrations are predictive of endoscopic recurrence in patients with Crohn's disease," Gut, vol. 50, no. 1, pp. 25-28, 2002.

[44] T. Yamamoto, S. Umegae, T. Kitagawa, and K. Matsumoto, "Mucosal cytokine production during remission after resection for Crohn's disease and its relationship to future relapse," Alimentary Pharmacology and Therapeutics, vol. 19, no. 6, pp. 671-678, 2004.

[45] T. Yamamoto, S. Umegae, T. Kitagawa, and K. Matsumoto, "Systemic and local cytokine production in quiescent ulcerative colitis and its relationship to future relapse: a prospective pilot study," Inflammatory Bowel Diseases, vol. 11, no. 6, pp. 589-596, 2005.

[46] Y. R. Mahida, M. Ceska, F. Effenberger, L. Kurlak, I. Lindley, and C. J. Hawkey, "Enhanced synthesis of neutrophilactivating peptide-I/interleukin-8 in active ulcerative colitis," Clinical Science, vol. 82, no. 3, pp. 273-275, 1992.

[47] L. Mazzucchelli, C. Hauser, K. Zgraggen et al., "Expression of interleukin- 8 gene in inflammatory bowel disease is related to the histological grade of active inflammation," American Journal of Pathology, vol. 144, no. 5, pp. 997-1007, 1994.

[48] J. O. Gebbers and H. F. Otto, "Immunohistochemical and electron microscopic observations on the local immune response in ulcerative colitis," Virchows Archiv-Abteilung A Pathologische Anatomie, vol. 374, no. 4, pp. 271-273, 1977.

[49] A. Keshavarzian, R. D. Fusunyan, M. Jacyno, D. Winship, R. P. MacDermott, and I. R. Sanderson, "Increased interleukin-8 (IL-8) in rectal dialysate from patients with ulcerative colitis: evidence for a biological role for IL-8 in inflammation of the colon," American Journal of Gastroenterology, vol. 94, no. 3, pp. 704-712, 1999.

[50] O. Watanabe, T. Ando, E. M. El-Omar et al., "Role of endoscopic ultrasonography in predicting the response to cyclosporin A in ulcerative colitis refractory to steroids," Digestive and Liver Disease, vol. 41, no. 10, pp. 735-739, 2009.

[51] M. J. Rosen, D. E. Moulton, T. Koyama et al., "Endoscopic ultrasound to guide the combined medical and surgical management of pediatric perianal Crohn's disease," Inflammatory Bowel Diseases, vol. 16, no. 3, pp. 461-468, 2010. 


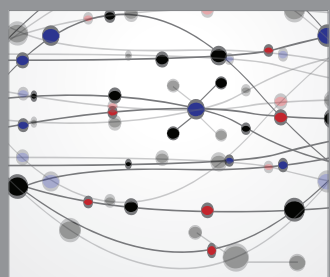

The Scientific World Journal
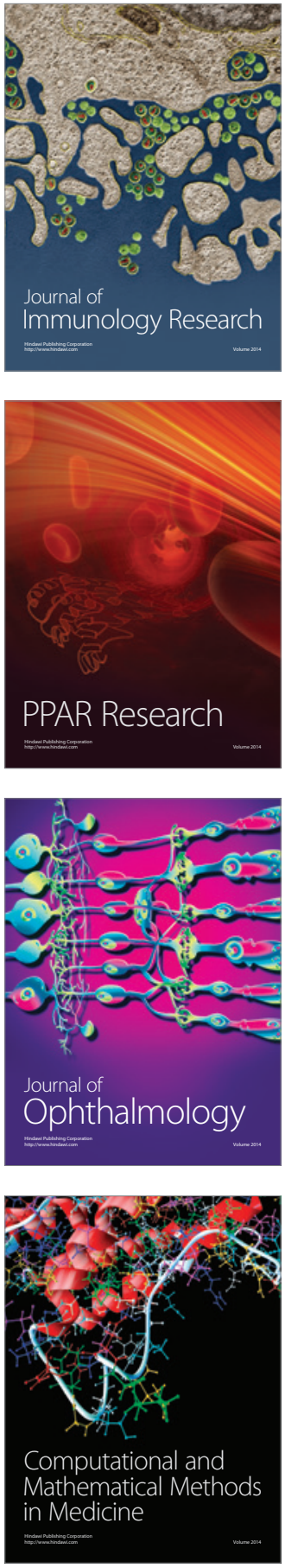

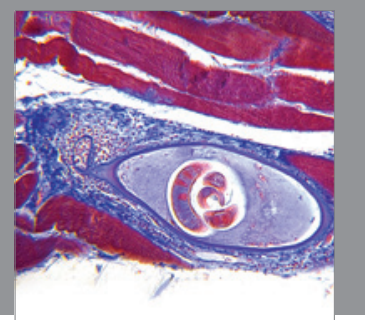

Gastroenterology

Research and Practice
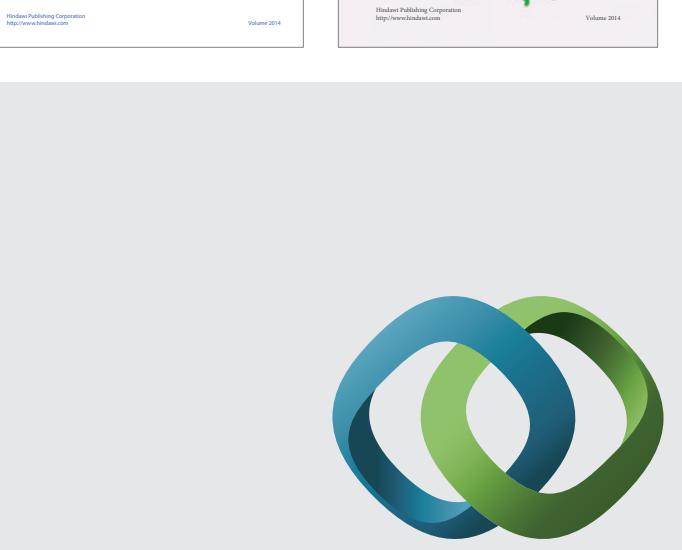

\section{Hindawi}

Submit your manuscripts at

http://www.hindawi.com
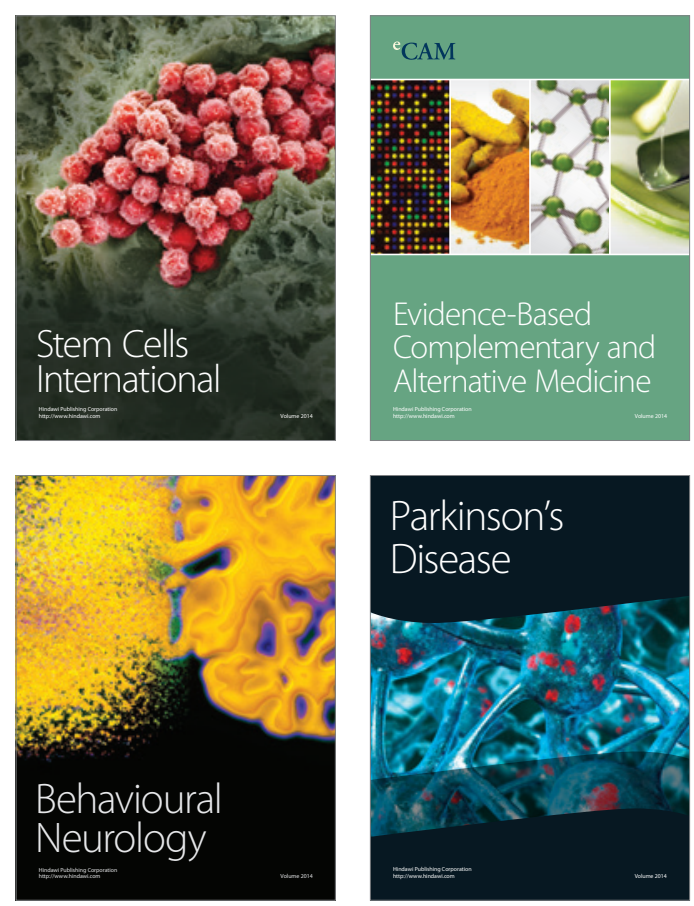



Journal of
Diabetes Research

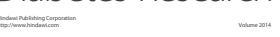

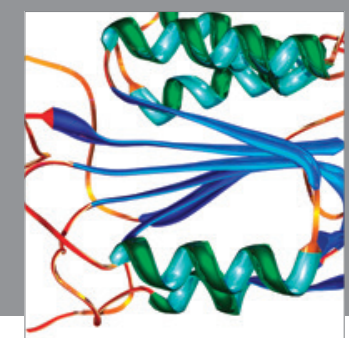

Disease Markers
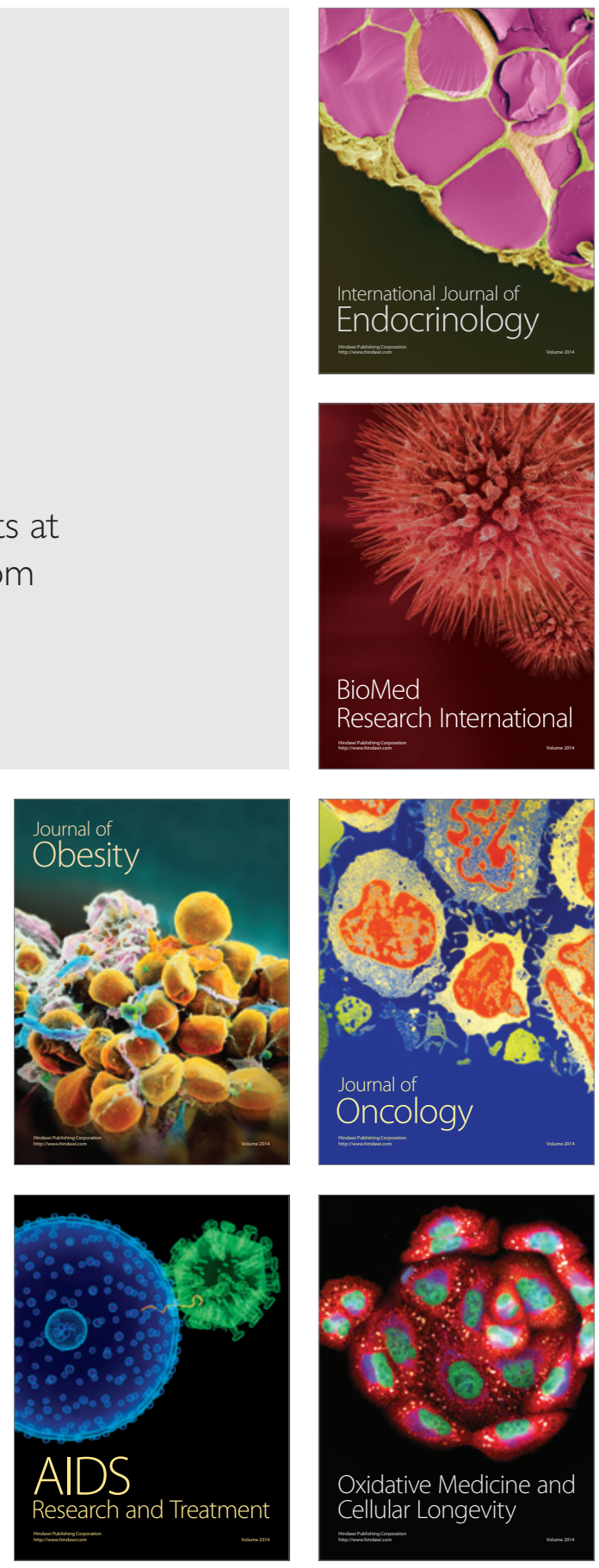